\title{
ENGINEERING THERMODYNAMICS
}




\section{ENGINEERING THERMODYNAMICS}

Theory, worked examples and problems

\section{G. Boxer}

Senior Tutor, Department of Mechanical Engineering,

University of Aston in Birmingham

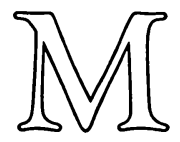


(C) G. Boxer 1976

All rights reserved. No part of this publication may be reproduced or transmitted, in any form or by any means, without permission

First published 1976 by

THE MACMILLAN PRESS LTD

London and Basingstoke

Associated companies in New York Dublin

Melbourne Johannesburg and Madras

ISBN 978-0-333-19226-9

ISBN 978-1-349-02828-3 (eBook)

DOI $10.1007 / 978-1-349-02828-3$

This book is sold subject to the standard conditions of the Net Book Agreement

The paperback edition of this book is sold subject to the condition that it shall not, by way of trade or otherwise, be lent, re-sold, hired out, or otherwise circulated without the publisher's prior consent in any form of binding or cover other than that in which it is published and without a similar condition including this condition being imposed on the subsequent purchaser 


\section{CONTENTS}

Preface $\quad$ ix

Nomenclature xiii

Some Useful Definitions and Analogies xv

system, boundary, state, property,

process, cycle, control surface,

control volume, work and heat

The Structure of Engineering Thermodynamics

xxiv interrelationship of the fundamental

laws with auxiliary information

A Fundamental Approach to the Solution of Thermodynamic Problems

$\mathrm{xxV}$

1 WORK AND HEAT TRANSFER

elementary applications of the sign

convention for work and heat transfers

2 WORK TRANSFER (HARDER EXAMPLES)

the calculation of work in certain

specified cases

3 FIRST LAW OF THERMODYNAMICS - SYSTEMS

the non-flow energy equation - internal

energy - heat and work transfers

4 FLUID PROPERTIES (EXCLUDING ENTROPY)

use of property tables to find values for

the five thermodynamic fluid properties

pressure, volume, temperature, internal

energy and enthalpy - interpolating in

tables and graphical explanation (gas

equation not treated at this stage)

5 SYSTEMS - MORE ADVANCED CALCULATIONS

the application of the non-flow energy equation together with the use of property tables and the characteristic gas equation

6 STEADY FLOW PROCESSES - VAPOURS

the steady-flow energy equation and the

use of property tables - throttling

calorimeter - nozzle-turbine 
7 STEADY FLOW PROCESSES - GASES

steady-flow energy equation and the

characteristic gas equation - mass

continuity equation

8 STEADY FLOW PROCESSES - ENERGY AND MOMENTUM

pressure and momentum thrust - jet pump or

ejector - rocket nozzle - turbo machine

9 UNSTEADY FLOW PROCESSES

unsteady-flow energy equation applied in

simple cases for both gases and vapours

10 GAS AND VAPOUR MIXTURES

Dalton's law - characteristic gas equation

applied to specific gases and mixtures -

the $\mathrm{kg}$-mol - mean values of $c_{p}, c_{V}, R$ and $\gamma$

- volumetric analysis and partial pressure -

use of first law of thermodynamics

11 STOICHIOMETRY

mass balance and molecular change for

reactants and products - stoichiometric

and non-stoichiometric combustion - analysis of

post combustion products - mixture strength

12 FIRST LAW APPLIED TO REACTING SYSTEMS

internal energy and enthalpy of formation and

of combustion - internal energy and enthalpy

of undissociated gases - non-flow energy and steady-flow energy equations in combustion -

effect of temperature on enthalpy of combustion

13 SECOND LAW OF THERMODYNAMICS - INTRODUCTORY CONCEPTS

heat engine and reversed heat engine -

efficiency and coefficient of performance

14 ENTROPY

reversible and irreversible forms of the first

law - statement of second law - entropy as a property and its use in solving real problems - isentropic efficiency and process - combined first and second laws - calculation of entropy

for gases and vapours - isentropic processes

for gases and vapours - nozzle efficiency -

the sense of steady flow and non-flow processes

by applying the second law when the first law

is of no help

15 VAPOUR POWER CYCLES

cycle efficiency - work ratio - specific fluid consumption - Rankine cycle - Carnot cycle for a vapour - throttling and superheating resuperheating - mixed pressure cycle - binary vapour cycle - pass out cycle 
16 GAS POWER CYCLES

a comparison of various idealised gas power

cycles with similar boundary conditions -

efficiency - work output - maximum and mean

effective pressures

17 AVAILABLE ENERGY

high and low grade energy - energy degradation

- non-flow and steady-flow availability -

irreversibility - ground or dead state -

effectiveness of a process - graphical

representation of availability - second law

analysis of power plant showing the

irreversibilities of various components 


\section{PREFACE}

The difficulty in preparing a tutorial volume on a subject like engineering thermodynamics is to select problems covering an adequate range of material and to the right depth which can be contained in a published form which is economically acceptable to all.

The content of this book is based on material taught to all first year mechanical engineering undergraduates at Aston university and is offered as a reasonably self-contained basis of fundamental work essential to any more advanced study of the subject.

The greatest benefit can be obtained by the student in the use of this book only by the exercise of considerable self-discipline. He or she should first attempt the given questions unaided and refer to the solutions for comparison and clarification only if difficulty arises. It is all too easy to read the question and solution together and convince oneself that the whole is fully understood.

It is only by approaching all questions as though they were set in an examination (with no help available) that one discovers one's weaknesses. Then it is possible to do something about it by further reading and further practice. This is the very essence of learning.

The teaching of engineering thermodynamics has changed radically in the last twenty five years or so mainly through the beneficial influence of Professor Keenan at Massachusetts Institute of Technology. It is vital to define all terms as rigorously as possible and an attempt to do this is made in the following pages.

A1l units are in the Système International d'Unites, or SI for short. It is perhaps right to mention here the dual practice with regard to the unit for pressure. Some authorities favour the use of the pascal $(\mathrm{Pa})$ i.e. $1 \mathrm{kN} / \mathrm{m}^{2}$, others the bar i.e. $10^{5} \mathrm{~N} / \mathrm{m}^{2}$ or $100 \mathrm{kN} / \mathrm{m}^{2}$. In this volume both are used since there is no clearcut all-round agreement.

I believe very firmly in the concept of one symbol for one physical idea and use $T$ for all temperatures (and let the units display whether a scale or thermodynamic-temperature is in use). Thus

(a) $T=20{ }^{\circ} \mathrm{C}$ is a scale temperature

(b) $T=293 \mathrm{~K}$ is a thermodynamic temperature.

However, a change in either a scale or a thermodynamic temperature is always given in degrees Kelvin.

The student must learn the vital importance of distinguishing between physical equations and numerical equations and in an effort to encourage this the Stroud convention is used throughout.

Anything multiplied by unity is unchanged and, for example, if a force of $1 \mathrm{~N}$ accelerates a mass of $1 \mathrm{~kg}$ by $1 \mathrm{~m} / \mathrm{s}^{2}$, then Newton says

$$
\begin{gathered}
\text { force }=\text { mass } \times \text { acceleration } \\
\text { or } \frac{1 \mathrm{~N}}{1 \mathrm{~kg} \times 1 \mathrm{~m} / \mathrm{s}^{2}}=\frac{\left[\mathrm{N} \mathrm{\textrm {s } ^ { 2 }}\right]}{[\mathrm{kg} \mathrm{m}]}=\text { UNITY }
\end{gathered}
$$


This unity bracket can be used as a multiplier to rationalise the units in a numerical equation.

For example, to obtain the temperature equivalent of a fluid velocity of $600 \mathrm{~m} / \mathrm{s}$, the following calculation is appropriate to air with $c_{p}=1.005 \mathrm{~kJ} /(\mathrm{kg} \mathrm{K})$.

$$
\begin{aligned}
\theta_{u} & =\text { Temperature equivalent of } u=u^{2} / 2 c_{p} \text { (physical equation) } \\
& =\frac{600^{2}}{2 \times 1.005} \frac{\mathrm{m}^{2}}{\mathrm{~s}^{2}} \frac{\mathrm{kg} \mathrm{K}}{\mathrm{kJ}}\left[\frac { \mathrm { N } \mathrm { s } \mathrm { s } ^ { 2 } ] } { [ \mathrm { kg } \mathrm { m } ] } \left[\frac{\mathrm{kJ}]}{[\mathrm{kNm}]}\right.\right. \text { (numerical equation) } \\
& =\frac{600^{2}}{2000 \times 1.005}=179.1 \mathrm{~K}
\end{aligned}
$$

Furthermore, for example, in calculating the power delivered from an engine which has a torque of $80 \mathrm{Nm}$ at a speed of rotation of 2000 $\mathrm{rev} / \mathrm{min}$

$$
\begin{aligned}
\text { Power } & =\text { rate of work transfer }=\text { torque } \times \text { angular velocity } \\
& =\tau \times \omega \quad \text { (physical equation) } \\
& \left.=80 \mathrm{Nm} \times 2000 \frac{\mathrm{rev}}{\mathrm{min}} \frac{[2 \pi \mathrm{rad}]}{\mathrm{rev}}\right]\left[\frac{\mathrm{min}}{60 \mathrm{~s}}\right]\left[\frac{\mathrm{kJ}}{1000 \mathrm{Nm}}\right] \\
& =16.76 \frac{\mathrm{kNm}}{\mathrm{s}}=16.76 \mathrm{~kW} \quad \text { (numerical equation) }
\end{aligned}
$$

The 1 ast three terms in this numerical equation are unity brackets and can be used whenever convenient. The use of such brackets obviates the need to worry about homogeneous units since a physical equation is valid in any system of units.

I have endeavoured to grade the problems in each chapter to cover a range of work from the most elementary undergraduate calculations to those appropriate to a first-year examination at University. Occasionally I have borrowed questions from London University B.Sc. Part I degree papers and am grateful to that institution for permission to do so whilst accepting sole responsibility for the solutions which are presented in this book.

The whole of engineering thermodynamics is based on the three fundamental principles of conservation of mass, energy and momentum and the concept of energy degradation embodied in the second law of thermodynamics. It is vital in the solution of all problems to find out just how these principles are brought into use.

It is equally vital to avoid splitting up the subject into purely artificial compartments with labels e.g. reciprocating compressors, gas turbines etc. and to endeavour to bracket certain formulae with each section.

To assist the student in formulating a logical approach to the solution of all problems in the subject I have laid out a set of fundamental questions to be answered each and every time until the right habits have been formed. In order to answer these questions correctly he will have to understand the basic definitions and the associated principles in the subject but once these are mastered he will find his approach is consistent and much more readily applicable than any arbitrary sectioning of the material with its demands upon pure memory as distinct from any appeal to logic.

The figure numbers are associated directly with the questions 
in which they occur and are not chronologically placed as in other texts. Thus figure 14.3 refers to question 3 in chapter 14 and is not necessarily the third figure in chapter 14 .

Throughout the book reference is made (in parentheses) to page numbers. The latter refer to tables of thermodynamic properties of fluids and other data by Y.R.Mayhew and G.F.C.Rogers published by Blackwell which should be used in conjunction with the solutions in this book.

I acknowledge with gratitude the help I have received from my colleagues at Aston over many years and particularly from Mr.F.R. Taylor who is responsible for a considerable proportion of the descriptive material at the start. 


\section{NOMENCLATURE}

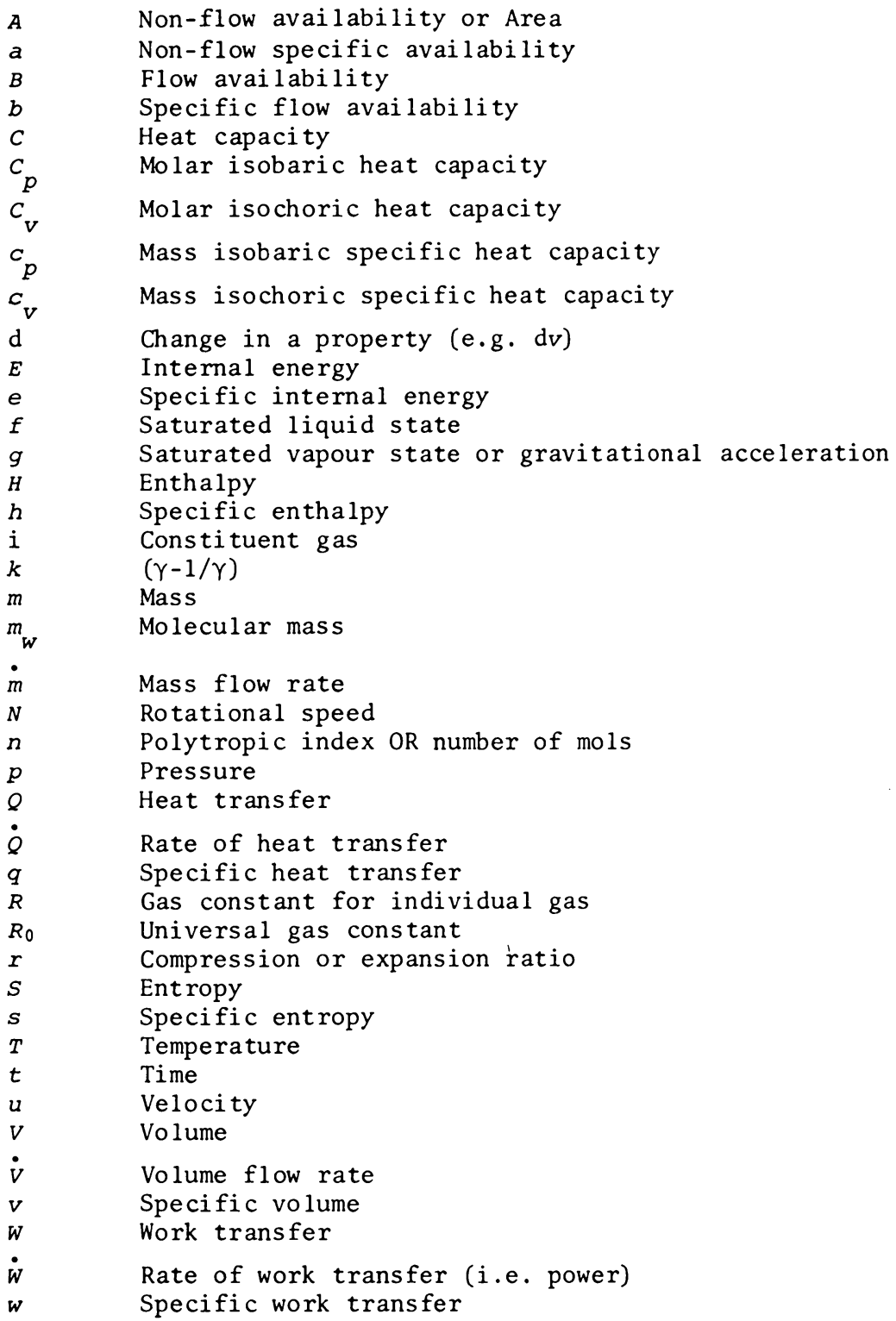


Quality of liquid/vapour mixture OR mole fraction

Mean value of $x$ (e.g.)

Datum height

$c_{p} / C_{v}=c_{p} / c_{V}$

Change in a non-property (e.g. $\delta Q)$

Angle OR change in temperature

Ratio of circumference to diameter of circle

Density

Efficiency

Effectiveness

Torque

$\omega \quad$ Angular Velocity

$\sum \quad$ Sum of

$£ \quad$ Sum of - round a cycle 


\section{SOME USEFUL DEFINITIONS AND ANALOGIES}

Historically, thermodynamics grew from the study of heat and temperature, which were often confused! Many of the terms and concepts used in thermodynamics, including heat, have been abstracted from common everyday usage where their meanings are very loosely defined. In the scientific study of thermodynamics precise definitions must be adopted and the thermodynamicist must accustom himself to using these terms in both contexts. Initially some self-discipline is necessary to adhere to the rigorous definitions used in thermodynamics.

Thermodynamics Thermodynamics may be described as the study of the equilibrium properties of large-scale systems in which temperature is an important variable.

System A prescribed and identifiable quantity of matter.

State The overall condition of a system at a given time.

Thermodynamic state That part of the overall condition of a system at rest which is dependent upon the state of motion and interaction of its component particles.

Property Any characteristic of a system which can be used to define its state i.e. which depends only upon the state of the system.

Extensive propertu A property whose magnitude is dependent upon the amount of matter considered e.g. volume.

Intensive property A property whose magnitude does not depend on the amount of matter considered. N.B. These are the potential properties e.g. pressure, temperature, electrical potential, etc. which tend to cause a change in state.

Specific property An extensive property expressed per unit quantity of matter. In the sense that this is independent of the mass in the system it may be regarded as an intensive property for the purpose of defining the state. Lower case letters are used for intensive properties and upper-case letters for extensive properties. e.g. a system of mass $m$ and volume $V$ has a specific volume $v=V / m$.

Equilibrium state A system is in equilibrium if its properties do not change in value when the system is isolated from its surroundings.

Thermodynamic equation of state Any mathematical relationship for 
the thermodynamic properties of a system in thermal equilibrium e.g. for a perfect gas the equation of state is $p v=R T$ where $R$ is a constant depending on the particular gas.

simple (or pure) substance A substance which is

(a) homogeneous in chemical composition

(b) homogeneous in chemical aggregation

(c) invariable in chemical aggregation.

N.B. such substances are important because the state of a system composed entirely of a pure substance may be completely defined by two independent, intensive properties.

Process A change of state of a system.

Cycle A process with identical initial and final states.

Phase of a substance The term phase is used to denote the molecular regime of a substance e.g. ice, water and steam are respectively the solid, liquid and vapour phases of $\mathrm{H}_{2} \mathrm{O}$.

Internal energy (E) The energy possessed by a system by virtue of the motion and arrangement of its molecules and the vibrations within the molecules. It is a property since its value depends only on the state of the system.

Work (W) Work is energy transferred across a system boundary by virtue of a potential property difference, other than temperature, across the boundary.

Both heat and work are fundamentally different from properties and are known as interactions because they are transient in nature. The nature of heat and work is dealt with in some detail in the following pages. However, the subject of thermodynamics is in essence the study of heat and work transfers upon the properties of a system and conversely the effect of a change in properties on the heat and work transfers.

It is vital to distinguish clearly between the properties of a fluid $(p, v, T$, etc.) and the interactions at the system boundary $Q$ and $W$ since the latter by their very nature can never be considered as properties. $Q$ and $w$ never exist IN a fluid - they occur at the boundary of a system and are transient in nature. To emphasise this point consider the following analogies.

Analogy I - Cloud/Lake

The figure depicts $\mathrm{H}_{2} \mathrm{O}$ in three forms.

(a) As vapour in the cloud.

(b) As liquid in the lake.

(c) As liquid falling from the cloud to the lake.

Now we describe the $\mathrm{H}_{2} \mathrm{O}$ in the cloud as WATER vapour, and that in the lake as WATER. However, we have a distinct name for the 
$\mathrm{H}_{2} \mathrm{O}$ in the act of passing between the two. We call it RAIN. The rain is not stored in the cloud nor in the lake - as soon as the rain reaches the 1 ake it becomes just water. The analogy is that this corresponds to the transfer of HEAT from a hot body to a cold

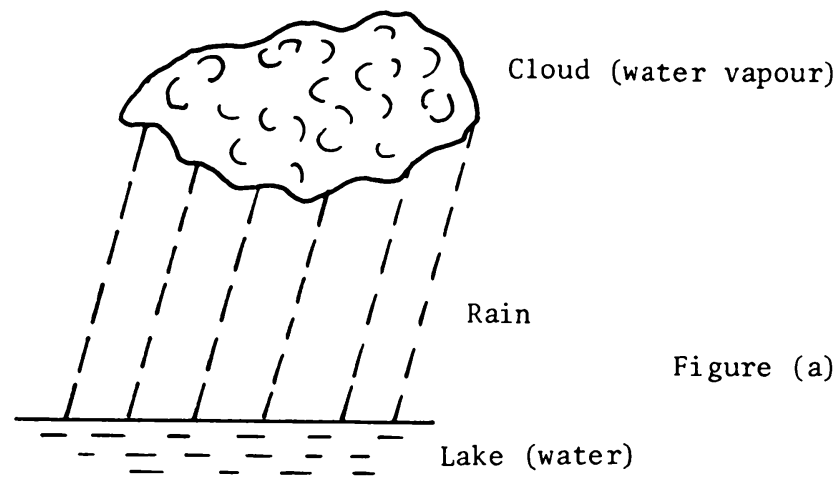

body wherein the hot body has high internal energy (relatively) and the cold body a lower internal energy. The heat transfer is also energy - BUT the internal energies are properties - the heat transfer is not. The internal energies correspond to the water stored respectively in the cloud and the lake and the heat transfer corresponds to the rain.

Analogy 2 - The Mountain

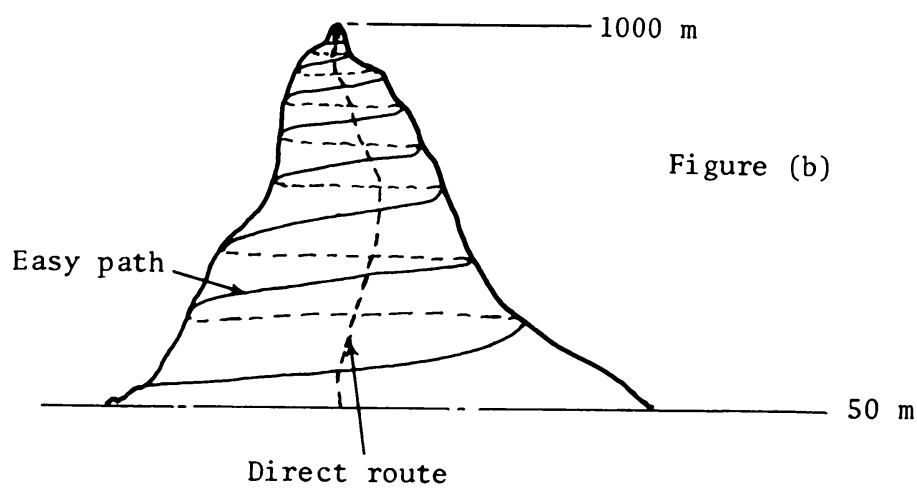

The figure depicts a mountain with the customary hazards facing any would-be climber. Assuming that the area has been properly surveyed, the height of the mountain summit above sea-level will be known (say $1000 \mathrm{~m}$ ) and let us suppose that a starting point for a climb is at say $50 \mathrm{~m}$ above sea-level.

Let us first assume that the climber is ambitious and attempts a climb by the most direct route. He will probably encounter scree slopes where he may slither around (we hope he is properly shod!) and if he knows what he is doing he will ultimately reach the top. The point is that when he gets there his overall change in height wi 11 be $1000 \mathrm{~m}-50 \mathrm{~m}$ i.e. $950 \mathrm{~m}$. 
Let us now assume purely for the sake of argument that this is a mountain with a well-worn and measured path which follows very easy gradients so that it is possible to walk up all the way without the considerable effort required in real mountaineering. When the top is reached the change in height is still $950 \mathrm{~m}$.

We should now realise that the values $50 \mathrm{~m}$ and $1000 \mathrm{~m}$ are PROPERTIES relating to the system (the mountain) and the PATH taken does not affect their values. However, the amount of work done by the person trying to reach the top depends very much on the path chosen and so does the amount of perspiration given off (i.e. the heat transfer). These latter cannot therefore be properties.

Digression on the expression 'change in' We have just used the expression change in height. The student is constantly involved in finding changes in quantities while studying mechanical engineering and many students do not understand this correctly.

Clearly we must know whether work is being delivered by a system or done on the system and whether heat is being added to a system or rejected from it. Thus sense plays a vital role. In other words $Q$ and $W$ have magnitude and sense (but not direction and are therefore not vectors).

Quite naturally as engineers we are interested in the production of work and it is logical to call work delivered positive work.

Since we most commonly achieve positive work from a system by first of all supplying heat to it then heat supplied is also treated as positive. Conversely work in to a system or heat rejected from a system are treated as negative.

It is vital to adhere to this scheme of things by interpreting changes in properties correctly.

In this sense I have found the bank-balance analogy useful as a means of getting the signs correct.

Suppose your bank balance on Monday was $£ 5$ and because of various demands you have an overdraft on the following Friday of say

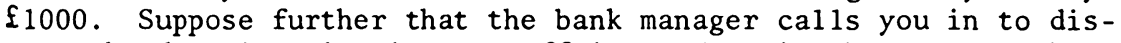
cuss the deterioration in your affairs. How will he present the case to you? The initial state of your balance was $+\mathfrak{f} 5$ (i.e. in credit) and the final state is $-£ 1000$ (overdraft).

Will he say that the change in your balance is

Initial - Final i.e. $+£ 5-£(-1000)=+£ 1005 ?$

Or will he say that the change in your balance is

Final - Initial i.e. $-£ 1000-£(+5)=-£ 1005$ ?

Clearly the latter is correct i.e.

change in means final - initial.

Thus for example the change in internal energy from state 1 to state 2 is $\left(E_{2}-E_{1}\right)$.

Control surface and control volume It is vital in the study of thermodynamics to distinguish between two kinds of process. 
(a) Non-flow process.

(b) Flow process.

To make this distinction we now define a control volume as any region in space having a fixed boundary and position. (Note that a system has a boundary which can change its shape and position but the mass contained therein is constant.)

We now define a non-flow process as one in which the system boundary change occurs wholly within the control volume under observation - i.e. no mass crosses the control surface which bounds the control volume.

Furthermore a flow process is one in which the system boundary change is such that mass does cross the control surface.

Note that $Q$ and $W$ can cross both system and control volume boundaries but by definition mass can only cross the latter.

Example of a non-flow process - expansion in a cylinder

\section{System boundary}

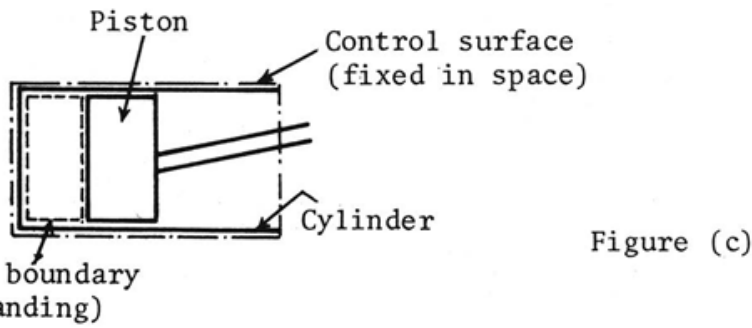

In the expansion depicted in the figure there is work transfer across the system boundary due to a pressure difference, there may be a heat transfer across the system boundary due to a temperature difference, but there is no mass transfer across the system boundary.

The same remarks apply exactly to the control surface depicted. Example of a flow process - flow through a nozzle

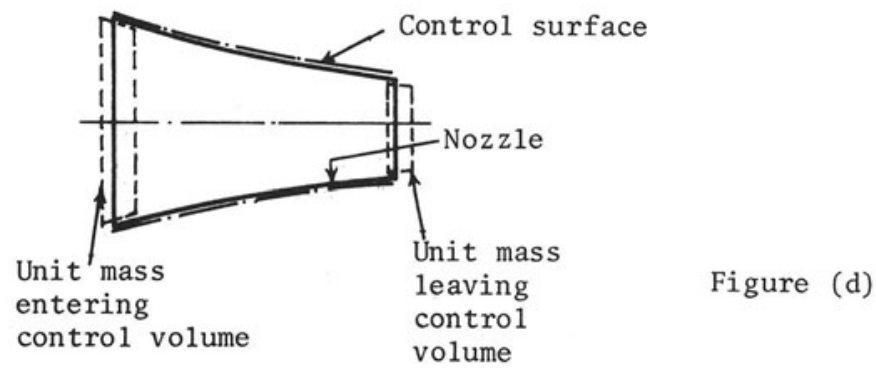

In this case there is no work transfer or heat transfer to consider but mass does cross the control surface even though by definition it does not cross the system boundary where in this case the system is taken to be unit mass of fluid passing through the nozzle. 
Work was previously defined as energy transferred across a system boundary by virtue of a potential property difference, other than temperature, across the boundary.

For example, electric currents represent transfers of energy due to electric potential (voltage) differences across a boundary. More importantly for mechanical engineers, movement of some part of a system boundary due to a force (or pressure difference) across the boundary is a typical example of work transfer.

The force and motion may be either perpendicular to the system boundary or tangential to it. Energy will be transferred as mechanical work provided that some component part of both force and motion act in the same direction.

It is now necessary to discuss the difference between controlled streamline flow and free, turbulent flow.

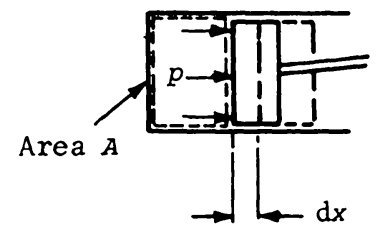

Figure (e)

Consider the expansion of a piston under the action of a fluid contained in a cylinder (see figure). The fluid is the system and its boundary is shown dotted.

Let the area of the piston face be $A$ and firstly let us assume the expansion to be so controlled that at a given moment the pressure everywhere in the fluid is $p$. The force on the piston face due to the fluid will be $p A$.

Now assume that after an infinitesimal lapse of time the piston has moved a distance $\mathrm{d} x$ to the right and the flow is so controlled that although the pressure has changed infinitesimally it is everywhere the same at all points in the fluid.

The work done during this piston movement by the fluid on the piston is the product of force and the distance moved

$$
\text { or } \begin{aligned}
\delta w & =(p A) \times \mathrm{d} x \\
& =p \mathrm{~d} V
\end{aligned}
$$

where $\mathrm{d} V$ is the volume swept out by the piston in this time.

Assuming the flow continues in this manner.

$$
w_{\text {overall }}=\int_{\text {initial state }}^{f i n a l ~ s t a t e}
$$

Conversely, as is the case in practice, when the flow is turbulent and free, there will be pressure gradients in the fluid at a given time and $p$ will not have a unique value at all points in the fluid at that time and nor will it be a unique function of $v$ during expansion.

Under these circumstances

$$
w \neq \int p d v
$$


The graphical interpretation of controlled, streamline flow is as shown in the figure.

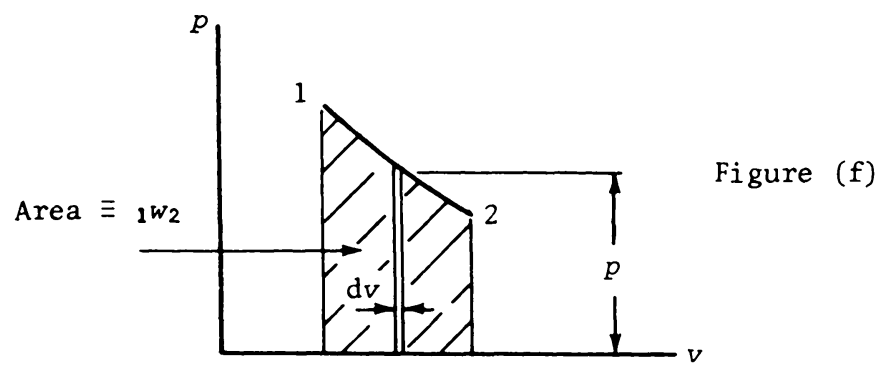

${ }_{1} W_{2} \equiv$ Area under the curve 1-2 which represents the controlled expansion where $p$ is some known function of $V$ and all intermediate states between 1 and 2 are known.

Conversely free, turbulent flow must be represented as below.

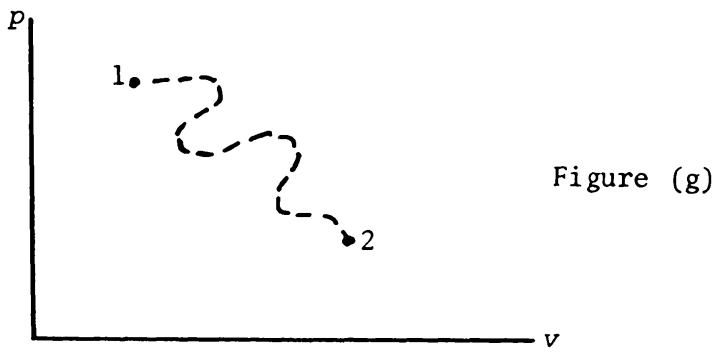

No area under a curve can be found since there is no known curve between 1 and 2. A state cannot be found between these two states because the fluid is never in equilibrium. In order to calculate $W$ in this case other information must be available. For example, from the first law of thermodynamics

$1 Q_{2}-{ }_{1} W_{2}=E_{2}-E_{1}$

Provided that the initial and final states 1 and 2 are known and also $1 Q_{2}$ then ${ }_{1} W_{2}$ is calculable.

The above arguments relate to a non-flow process if the control volume just embraces the entire cylinder.

However, many students cannot distinguish between this work transfer and that corresponding to a flow process made up of three distinct parts, namely suction, expansion and exhaust.
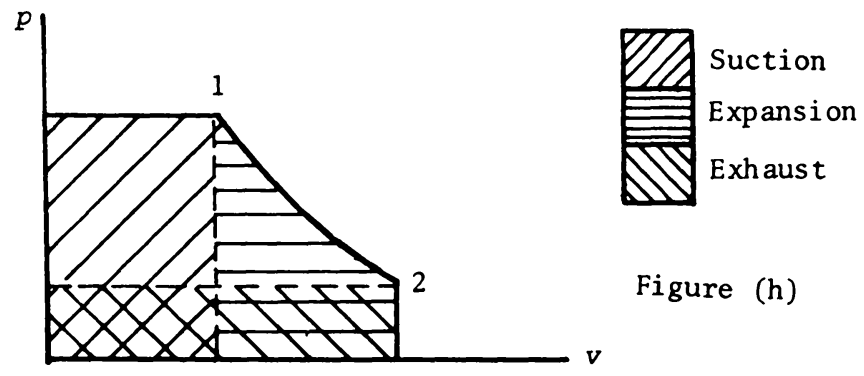

Figure (h) 
Thus for the same cylinder but now assuming a constant pressure suction process to start with, the same expansion as before and a constant pressure exhaust process to finish with we have the configuration depicted as shown in the above $p-V$ diagram.

The total work done is given by the work of suction (positive because fluid does work on the crank) plus the work of expansion as before plus the work of exhaust (negative since the crank does work on the fluid in this case).

$$
\begin{aligned}
& \text { Thus } w=w_{\text {suction }}+w_{\text {expansion }}+w_{\text {exhaust }} \\
& \text { or } \quad w=p_{1} V_{1}+\int_{V_{1}}^{V_{2}} \mathrm{~d} V-p_{1} v_{2}
\end{aligned}
$$

This work is clearly given by

$$
W=\int_{p_{2}}^{p_{1}} V \mathrm{~d} p
$$

which is the total work of suction, expansion and exhaust and is represented by the area shown shaded in the figure.

The distinction between $\int p \mathrm{~d} V$ work and $\int V \mathrm{~d} p$ work is very important and further consideration will reveal that the terms $p_{1} V_{1}$ and $p_{2} V_{2}$ represent the energy transfers associated with the movement of the system (a given packet of fluid in a given time) into and out of the control volume (the cylinder).

- One should note that these latter energy transfers take place with no change in the thermodynamic state of the fluid in the system - they are there simply because mass is being transferred into and out of the control volume. However, work transfer inevitably occurs with these mass transfers.

The thermodynamic state changes from 1 to 2 when a fixed packet of fluid contained within the control volume undergoes an expansion process.

\section{The nature of heat transfer}

Heat is energy transferred across a system boundary by virtue of a temperature difference across the boundary.

For example consider block $A$ at a uniform temperature $T_{A}$ and block $B$ at another uniform temperature $T_{B}$ (where $T_{B}<T_{A}$ ). ${ }^{A}$ Let the two be brought into contact and be thermally insulated from their surroundings but not from each other. Energy will flow from A to $B$ because of the temperature difference $T_{A}-T_{B}$. This will cause a reduction in molecular activity (and hence internal energy) of block $A$ and a corresponding increase in molecular activity (and hence internal energy) of block $B$.

The choice of a system boundary is quite arbitrary. Consider in the first case that block $A$ is the system and that block $B$ forms part of the surroundings. The energy transfer, due to the temperature difference, takes place across a system boundary and is thus termed heat according to the above definition (see figure) 


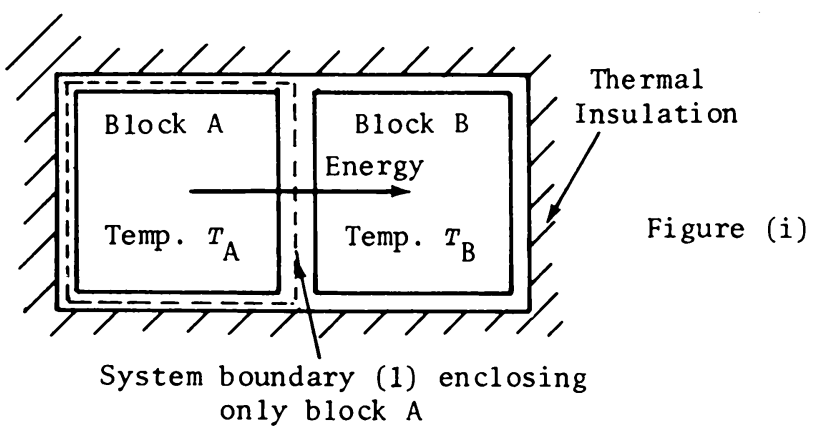

By choosing the system boundary to include both blocks A and B the energy transfer no longer crosses a system boundary and would not be termed heat relative to the new system.

This second system is not in thermal equilibrium since it changes state by redistribution of its internal energy although it is isolated from its surroundings (see figure).

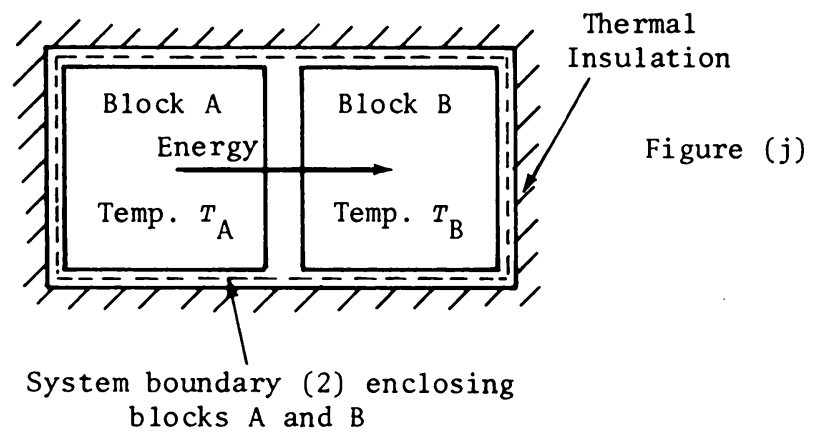

Heat transfer is a subject in its own right and can occur in various modes, conduction, convection and radiation or a combinattion of one or more of these. We are only interested here in the numerical value of heat as predicted by the application of the laws of thermodynamics to systems and not in the detailed mechanism of the energy transfer. 


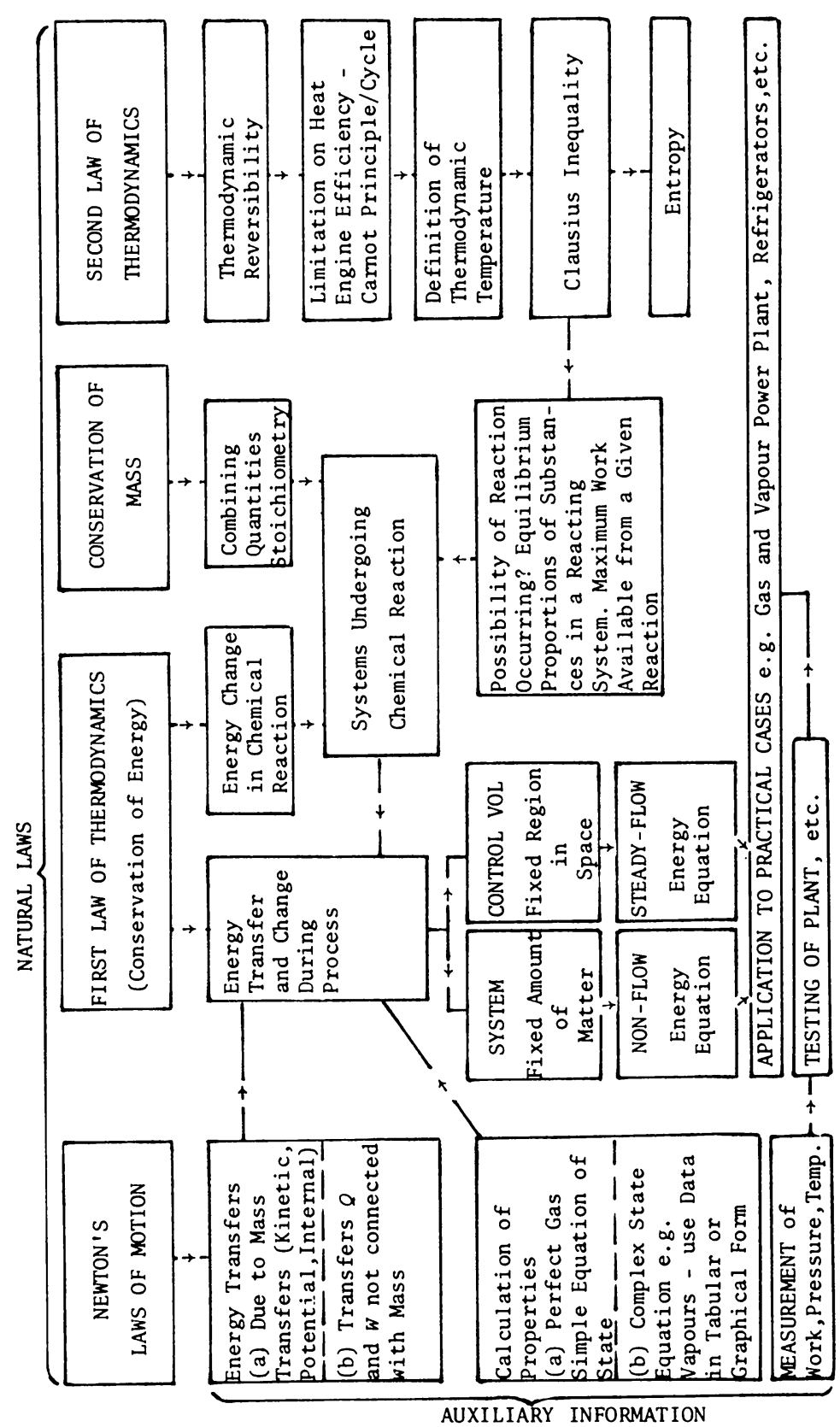

THE STRUCTURE OF ENGINEERING THERMODYNAMICS

This diagram is intended to show how the various topics in the subject are inter-related. Note that not all the topics shown are dealt with in a first year and some details are omitted. 


\section{A FUNDAMENTAL APPROACH TO THE SOLUTION OF THERMODYNAMICS PROBLEMS}

The following is an attempt to assist students in adopting a rational approach to the solution of all problems in thermodynamics. If the following questions are correctly answered each and every time a problem is attempted there should be no fundamental difficulty in obtaining a solution.

1. What kind of process? (a) Flow or (b) Non-flow?

To answer this it is necessary to define the system boundary and/or the control surface and then decide whether mass crosses the latter.

2. What kind of fluid?
(a) Liquid?
(b) Liquid/Vapour Mixture?
(c) Saturated Vapour?
(d) Superheated Vapour?
(e) Perfect Gas?
(f) Semi-perfect Gas? (i.e. one with variable $c_{p}$ and $c_{v}$ )

3. Have you drawn a state diagram(s)?

It is vitally important to get a picture of events.

4. Which form of the energy equation is required?
(a) Non-flow?
(b) Steady-flow?
(c) Unsteady-flow?

5. Do you need to use the mass continuity equation?

$\dot{m} v=u A$

6. Do you need to use the momentum equation?

7. Have you used the correct language? e.g.

$w$ in $\mathrm{kJ} / \mathrm{kg}, w$ in $\mathrm{kJ}, \dot{w}$ in $\mathrm{kW}$, etc.

8. Always put in dimensions with the numbers in your reasoning.

Dimensions are a check on your arguments and are more important than the numbers in front. Use unity brackets to rationalise the dimensions.

9. Do not effect numerical calculation until you have to. 